\title{
MEMETIC ALGORITHM FOR THE NURSE SCHEDULING PROBLEM
}

\author{
Tad Gonsalves and KoheiKuwata \\ Department of Information and Communication Sciences, \\ Faculty of Science \& Technology, \\ Sophia University, 7-1 Kioicho, Chiyoda-ku, Tokyo, 102-8554 Japan
}

\begin{abstract}
The Nurse Scheduling Problem (NSP), like the well-known Travelling Salesman Problem (TSP), is an NP-hard problem. In this study, we use a tailor-made meta-heuristic Memetic Algorithm (MA) to optimize the NSP. The MAis a hybrid algorithm, being a combination of the Genetic Algorithm (GA) and a local search algorithm. The performance of the MA is found to be superior to that of a solitary algorithm like GA. The MA solves the NSP in two stages. In the first stage, the randomly generated solutions are evolved till they become feasible (i.e., the hard constraints are satisfied) and in the second stage, these solutions are further evolved so as to minimize the violations of the soft constraints. In the final stage, the MA produces optimal solutions in which the hard as well as the soft constraints are completely satisfied.
\end{abstract}

\section{Introduction}

Many nurse scheduling problems are described in the literature and most papers focus on the solution techniques [7], [8], [21], [38]. The nurse scheduling problem (NSP) deals with the task of creating weekly or monthly schedules for hospital wards by assigning a feasible shift pattern to each nurse. The schedules, while satisfying the working contracts of the nurses as constraints, has to meet the demand for a given number of nurses of different grades on each shift. The schedule also has to be fair to all the nurses by satisfying their preferences as much as possible and by evenly distributing the unpopular shifts [3].

The NSP has traditionally been solved using the Integer Programming Techniques[17],[20], [40], Tabu search [14], [15],[37], and heuristic methods [1], [11], [23]. Simulation is also effectively used in some studies [9],[10]. Some of the recent AI techniques include the use of Simulated Annealing [19], [30], Genetic Algorithm [2],[16],[24],[39], co-operative Genetic algorithm [3436],Artificial Immune System [28] and different versions of Evolutionary Algorithms [4],[6],[22].The conventional GA does not yield satisfactory solutions [2].Therefore, we believe that a hybrid methodology involving an Evolutionary Algorithm that finds several feasible solutions and a Local Search exploiting the inherent knowledge of the problem to optimize the intermediate feasible solutions is an appropriate tool to tackle this highly complex problem.

The hybridization of evolutionary algorithms (EAs) with other techniques can greatly improve the efficiency of search [12], [18]. EAs hybridized with local search techniques are named as Memetic Algorithms [26],[29],[31-33].A common approach is to apply the local search to the GA population after crossover and mutation, with the aim of exploiting the best search regions. An important aspect concerning MAs is the trade-off between the exploration abilities of the EA and the exploitation abilities of the local search technique [25]. 
In this study, we use a Memetic Algorithm (MA) to solve the complex nurse scheduling problem. The MA algorithm is a hybrid of the Genetic Algorithm (GA) and Local Search (LS). The GA follows a simple coding scheme and after the recombination operations, LS is applied using problem-specific knowledge. A number of random shift schedules are generated. Penalties are imposed for the violation of the hard as well as the soft constraints of the shift schedules. The MA solves the problem in two phases. In the first phase, it tries to resolve all the violations of the hard constraints. This leads to feasible solutions. In the second phase, MA works with the feasible solution and further evolves them eliminating, or at least minimizing the soft constraints. The result is optimal solutions satisfying the management requirements as well as the nurses' preferences.

This paper is organized as follows: In section2, we explain the general NSP and a specific version that we have solved. In section3, we describe in detail the design and application of a tailor-made MA to solve the NSP with the obtained results. We conclude the study in section 4 .

\section{Nurse Scheduling Problem (NSP)}

There are many versions of the NSP found in literature. However, there exists neither a standard version nor benchmark problems to test new solution techniques [7]. In this study, we consider the nurse scheduling problem and the datasets provided by the Queen's Medical Centre (QMC) in Nottingham, UK. Our motivation to adopt this problem is that the problem is well-formulated and made freely accessible online with its associated data[27].

In the QMC nurse scheduling problem, a medium-sized group of nurses (20-30) should be scheduled over a planning period of four weeks. The daily schedule consists of the following three shifts: early (E; 7:00 14:45), late (L; 13:30 21:15) and night (N; 21:00 7:15) each with different coverage demand. The schedule should simultaneously satisfy the coverage demand and the working regulations constraints, as well as the nurses' preferences as far as possible. There are 6 hard constraints (must be satisfied) and 6 soft constraints (should be satisfied) which are listed below.

\section{Hard Constraints}

OneShiftADay: A nurse can work only one shift per day.

MaxHours: Nurses work a maximum number of hours.

MaxDaysOn : Nurses work a maximum number of consecutive days without a break.

MinDaysOn : Nurses work a minimum number of consecutive days.

Succession : Several shift combinations are not allowed ,e.g. night shift followed by early shift and vice-versa.

Hard Request :Nurses must take the annual leave they are entitled to.

\section{$\underline{\text { Soft Constraints }}$}

SoftRequest: Nurses prefer some favorable shifts (or days-off) for some days once in a while.

SingleNight: Nurses prefer to work night shifts in blocks of two or more.

WeekendBalance: Nurses should not work more than 3 out of 4 consecutive weekends. WeekendSplit: As for the weekends, the nurses prefer to work both days of the weekend or take both the days of the weekend off.

Coverage: The coverage demand for each shift should be satisfied as closely as possible.

CoverageBalance: The deficit/surplus of coverage demand should be balanced for all shifts in the planning period. 
International Journal of Artificial Intelligence \& Applications (IJAIA) Vol. 6, No. 4, July 2015

Nurses in QMC are employed either as full-time or part-time. Full-time nurses are required to work at most 75 hours per fortnight while part-time nurses work for lesser number of hours. They belong to one of the four possible qualification categories. Registered (RN), Enrolled (EN), Auxiliary (AN) and Student (SN). RNs and ENs are classified as qualified (QN) while QNs and ANs are both employed (PN).

Table 1. The cover requirements of nurses with different qualifications

\begin{tabular}{|l|c|c|c|}
\hline & Early & Late & Night \\
\hline QNs & 4 & 3 & 2 \\
\hline RNs & 1 & 1 & 0 \\
\hline ETs & 1 & 1 & 1 \\
\hline
\end{tabular}

\section{NSP optimization using MA}

The Memetic Algorithm (MA) we have used to solve the above QMC NSP consists of the Genetic Algorithm combined with a local search. The MA flowchart is shown in Fig. 1. It describes in detail each of the steps of the MA.

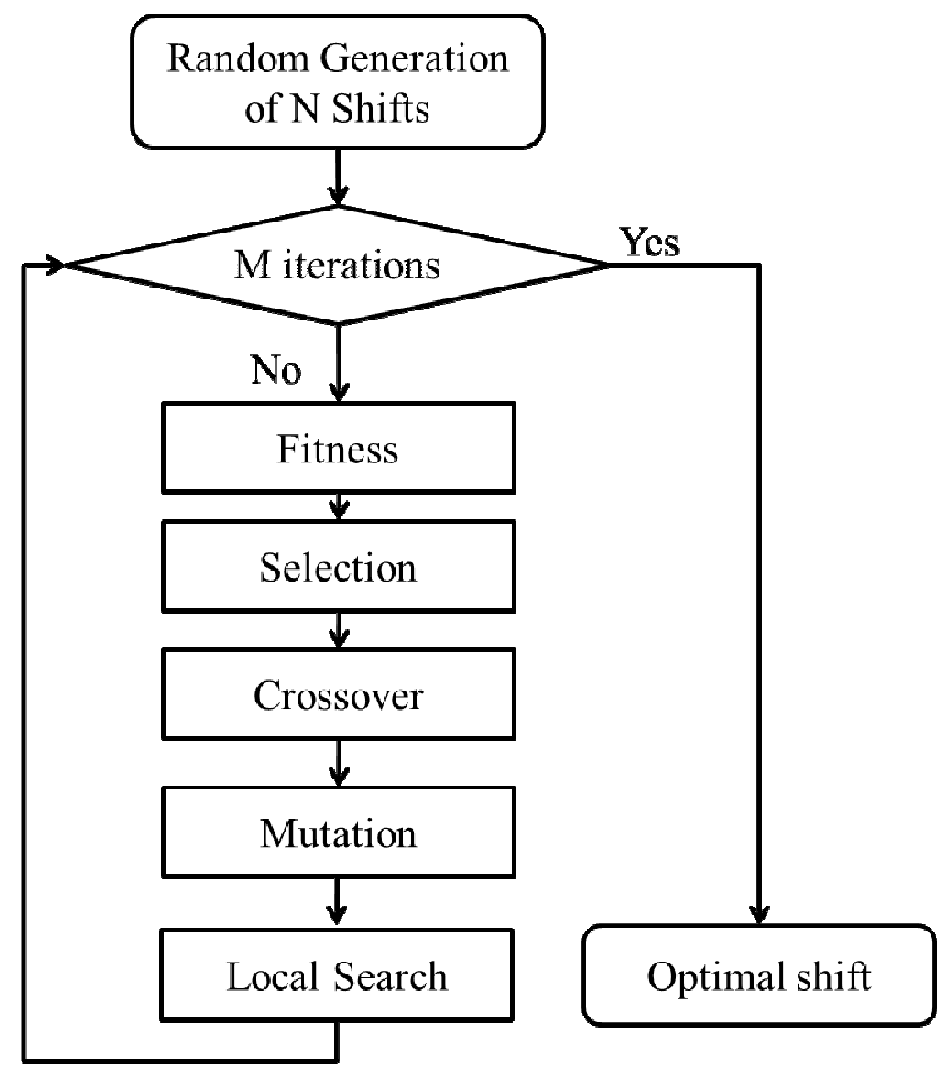

Fig.1.Memetic Algorithm flowchart

Step 1: Random generation of shift tables 
$\mathrm{N}$ number of shifts tables is generated randomly. A typical shifts table created according to the preferences of the nurses is shown in Table 2. The meanings of the entries in the table are explained below the table. The MA coding shown in Table 3 is as follows: 0 implies a free day while 1 implies a particular shift for a given nurse. 4 implies that the nurse does not want to work on that particular shift and 5 implies the annual leave. The real-number coding does not follow the canonical bit-string GA. The advantage is that it avoids the computational overhead brought about by the genotype-phenotype conversion.

Table 2. Shifts according to the nurses' preferences

\begin{tabular}{|c|c|c|c|c|c|c|c|c|c|c|c|c|c|c|c|c|c|c|c|c|c|c|c|c|c|c|c|c|}
\hline & 5 & 6 & 7 & 8 & 9 & 10 & 11 & 12 & 13 & 14 & 15 & 16 & 17 & 18 & 19 & 20 & 21 & 22 & 23 & 24 & 25 & 26 & 27 & 28 & 29 & 30 & 31 & 1 \\
\hline & $\mathrm{M}$ & $\mathrm{T}$ & $\mathrm{W}$ & $\mathrm{Th}$ & $\mathrm{F}$ & $\mathrm{S}$ & $\mathrm{Su}$ & M & $\mathrm{T}$ & $\mathrm{W}$ & Th & $\mathrm{F}$ & $S$ & $\mathrm{Su}$ & $\mathrm{M}$ & $\mathrm{T}$ & $\mathrm{W}$ & Th & $\mathrm{F}$ & $S$ & $\mathrm{Su}$ & $\mathrm{M}$ & $T$ & W & Th & $\mathrm{F}$ & $\mathrm{S}$ & $\mathrm{Su}$ \\
\hline Anita & & & E & $\mathrm{O}$ & $\mathrm{O}$ & $\mathrm{L}$ & $\mathrm{E}$ & & & & E & E & $\mathrm{O}$ & $\mathrm{O}$ & AL & $\mathrm{AL}$ & $\mathrm{AL}$ & $\mathrm{AL}$ & $\mathrm{AL}$ & $\mathrm{AL}$ & $\mathrm{AL}$ & $\mathrm{AL}$ & $\mathrm{AL}$ & $\mathrm{L}$ & E & $\mathrm{N}$ & $\mathrm{N}$ & $\mathrm{O}$ \\
\hline Cheryl & & $\mathrm{AL}$ & $\mathrm{L}$ & & & $\mathrm{O}$ & $\mathrm{O}$ & & $\mathrm{AL}$ & & $\mathrm{AL}$ & & & & $\mathrm{AL}$ & $\mathrm{AL}$ & $\mathrm{AL}$ & $\mathrm{AL}$ & $\mathrm{AL}$ & $\mathrm{AL}$ & $\mathrm{AL}$ & $\mathrm{L}$ & & & & & $\mathrm{O}$ & $\mathrm{O}$ \\
\hline Chris & & $\mathrm{E}$ & $\mathrm{L}$ & & $\mathrm{E}$ & & $\mathrm{N}$ & & $\mathrm{L}$ & $\mathrm{L}$ & $\mathrm{AL}$ & $\mathrm{E}$ & $\mathrm{L}$ & & $\mathrm{O}$ & $O$ & $O$ & $\mathrm{O}$ & 0 & 0 & 0 & $\mathrm{O}$ & $\mathrm{O}$ & $\mathrm{O}$ & 0 & $\mathrm{O}$ & $\mathrm{O}$ & $\mathrm{O}$ \\
\hline Claire & $\mathrm{O}$ & $\mathrm{O}$ & & $\mathrm{AL}$ & & & & & & $\mathrm{E}$ & $\mathrm{AL}$ & $\mathrm{AL}$ & & & $\mathrm{L}$ & & & & & & & & & & & & & \\
\hline Daryl & $\mathrm{O}$ & $\mathrm{O}$ & O & & $\mathrm{L}$ & $\mathrm{L}$ & $\mathrm{O}$ & $\mathrm{L}$ & O & $\mathrm{L}$ & $\mathrm{AL}$ & $\mathrm{AL}$ & $\mathrm{AL}$ & $\mathrm{N}$ & $\mathrm{O}$ & $\mathrm{O}$ & L & $\mathrm{O}$ & $\mathrm{L}$ & $\mathrm{L}$ & AL & $\mathrm{AL}$ & $\mathrm{AL}$ & AL & $\mathrm{AL}$ & $\mathrm{AL}$ & $\mathrm{AL}$ & $\mathrm{AL}$ \\
\hline Julie P & $\mathrm{O}$ & E & L & E & $\mathrm{O}$ & $\mathrm{O}$ & $\mathrm{L}$ & $\mathrm{O}$ & $\mathrm{O}$ & $\mathrm{N}$ & $\mathrm{N}$ & $\mathrm{O}$ & $\mathrm{O}$ & $\mathrm{O}$ & $\mathrm{O}$ & E & L & E & $\mathrm{O}$ & $\mathrm{O}$ & L & O & E & E & AL & $\mathrm{O}$ & $\mathrm{O}$ & $\mathrm{O}$ \\
\hline Kriska & & & & & & & & & & & & $\mathrm{E}$ & $\mathrm{O}$ & $\mathrm{O}$ & $\mathrm{L}$ & $\mathrm{AL}$ & $\mathrm{AL}$ & $\mathrm{AL}$ & $\mathrm{AL}$ & $\mathrm{AL}$ & $\mathrm{AL}$ & $\mathrm{AL}$ & & & & & & \\
\hline Linda B & $\mathrm{L}$ & E & E & E & E & $\mathrm{O}$ & $\mathrm{O}$ & $\mathrm{N}$ & $\mathrm{N}$ & $\mathrm{O}$ & E & AL & $\mathrm{O}$ & $\mathrm{O}$ & L & E & $\mathrm{O}$ & $\mathrm{O}$ & L & $\mathrm{L}$ & L & E & E & E & $\mathrm{O}$ & O & E & $\mathrm{L}$ \\
\hline Linda W & & & & & & & & & & & & & & & $\mathrm{AL}$ & $\mathrm{AL}$ & $\mathrm{AL}$ & $\mathrm{AL}$ & $\mathrm{AL}$ & & & & & & & $\mathrm{O}$ & & \\
\hline $\mathrm{Liz}$ & & & & & & $\mathrm{O}$ & $\mathrm{O}$ & & $\mathrm{O}$ & & & & $\mathrm{O}$ & $\mathrm{O}$ & & & & & & & & & & & & & & \\
\hline Louise & $\mathrm{L}$ & & & & & & & & & & & & & & & & & & & & & & & $\mathrm{N}$ & $\mathrm{N}$ & & & \\
\hline Malinka & $\mathrm{L}$ & & $\mathrm{L}$ & $\mathrm{L}$ & $\mathrm{E}$ & $\mathrm{O}$ & $\mathrm{O}$ & $\mathrm{O}$ & $\mathrm{L}$ & $\mathrm{O}$ & $\mathrm{E}$ & $\mathrm{L}$ & $\mathrm{L}$ & $\mathrm{E}$ & $\mathrm{L}$ & $\mathrm{L}$ & & $\mathrm{L}$ & $\mathrm{O}$ & $\mathrm{O}$ & $\mathrm{E}$ & $\mathrm{L}$ & $\mathrm{L}$ & $\mathrm{AL}$ & $\mathrm{AL}$ & $\mathrm{AL}$ & $\mathrm{O}$ & $\mathrm{O}$ \\
\hline Margaret & $\mathrm{O}$ & $\mathrm{L}$ & & $\mathrm{L}$ & E & $\mathrm{O}$ & $\mathrm{O}$ & $\mathrm{L}$ & E & & $\mathrm{O}$ & $\mathrm{O}$ & $\mathrm{O}$ & $\mathrm{L}$ & E & L & $\mathrm{N}$ & $\mathrm{N}$ & L & $\mathrm{O}$ & 0 & O & & & & & & \\
\hline Nicola & $\mathrm{E}$ & $\mathrm{O}$ & $\mathrm{E}$ & $\mathrm{O}$ & $\mathrm{N}$ & $\mathrm{N}$ & $\mathrm{E}$ & $\mathrm{O}$ & $\mathrm{O}$ & $\mathrm{O}$ & $\mathrm{O}$ & $\mathrm{L}$ & $\mathrm{O}$ & $\mathrm{E}$ & E & $\mathrm{O}$ & $\mathrm{O}$ & $\mathrm{O}$ & $\mathrm{L}$ & $\mathrm{O}$ & $\mathrm{E}$ & $\mathrm{E}$ & $\mathrm{O}$ & 0 & $\mathrm{O}$ & $\mathrm{L}$ & $\mathrm{O}$ & $\mathrm{E}$ \\
\hline Nynke & $\mathrm{O}$ & $\mathrm{O}$ & $\mathrm{O}$ & $\mathrm{O}$ & $\mathrm{O}$ & $\mathrm{O}$ & $\mathrm{L}$ & $\mathrm{E}$ & $\mathrm{L}$ & $\mathrm{O}$ & $\mathrm{O}$ & $\mathrm{O}$ & $\mathrm{O}$ & $\mathrm{O}$ & $\mathrm{N}$ & $\mathrm{N}$ & O & $\mathrm{O}$ & $\mathrm{O}$ & 0 & $\mathrm{O}$ & $\mathrm{O}$ & $\mathrm{O}$ & $\mathrm{O}$ & $\mathrm{O}$ & $\mathrm{O}$ & $\mathrm{O}$ & $\mathrm{O}$ \\
\hline Sue E & $\mathrm{N}$ & $\mathrm{N}$ & $\mathrm{O}$ & $\mathrm{O}$ & $\mathrm{O}$ & $\mathrm{L}$ & $\mathrm{L}$ & $\mathrm{L}$ & $\mathrm{E}$ & L & E & $\mathrm{O}$ & $\mathrm{O}$ & $\mathrm{O}$ & $\mathrm{L}$ & E & $\mathrm{L}$ & $\mathrm{L}$ & $\mathrm{O}$ & $\mathrm{O}$ & $\mathrm{O}$ & $\mathrm{L}$ & E & $\mathrm{O}$ & $\mathrm{O}$ & $\mathrm{O}$ & $\mathrm{L}$ & $\mathrm{L}$ \\
\hline Susan & $\mathrm{O}$ & $\mathrm{L}$ & $\mathrm{E}$ & E & $\mathrm{O}$ & $\mathrm{O}$ & $\mathrm{O}$ & $\mathrm{AL}$ & $\mathrm{AL}$ & $\mathrm{AL}$ & $\mathrm{O}$ & $\mathrm{O}$ & $\mathrm{O}$ & $\mathrm{O}$ & $\mathrm{AL}$ & $\mathrm{O}$ & $\mathrm{O}$ & $\mathrm{O}$ & E & E & E & $\mathrm{N}$ & $\mathrm{N}$ & $\mathrm{O}$ & 0 & $\mathrm{O}$ & $\mathrm{O}$ & $\mathrm{O}$ \\
\hline Tess & $\mathrm{O}$ & $\mathrm{O}$ & $\mathrm{AL}$ & & $\mathrm{L}$ & $\mathrm{O}$ & $\mathrm{L}$ & $\mathrm{E}$ & & $\mathrm{O}$ & & & $\mathrm{O}$ & $\mathrm{O}$ & & $\mathrm{O}$ & & & 0 & $\mathrm{O}$ & $\mathrm{L}$ & E & $\mathrm{O}$ & & & & $\mathrm{O}$ & $\mathrm{O}$ \\
\hline Tricia & $\mathrm{E}$ & $\mathrm{E}$ & $\mathrm{N}$ & $\mathrm{N}$ & $\mathrm{O}$ & $\mathrm{O}$ & $\mathrm{E}$ & $\mathrm{E}$ & & E & $\mathrm{L}$ & $\mathrm{E}$ & $\mathrm{O}$ & $\mathrm{O}$ & $\mathrm{E}$ & E & E & $\mathrm{E}$ & $\mathrm{E}$ & 0 & 0 & & $\mathrm{E}$ & $\mathrm{E}$ & $\mathrm{L}$ & $\mathrm{L}$ & $\mathrm{O}$ & $\mathrm{O}$ \\
\hline
\end{tabular}

The symbols in the above data represent the following: E(Early: 7:00-14:30); L(Late: 13:0021:15; N(Night: 21:00-7:15); O (Free day); AL (Annual leave)

Table 3. MA coding

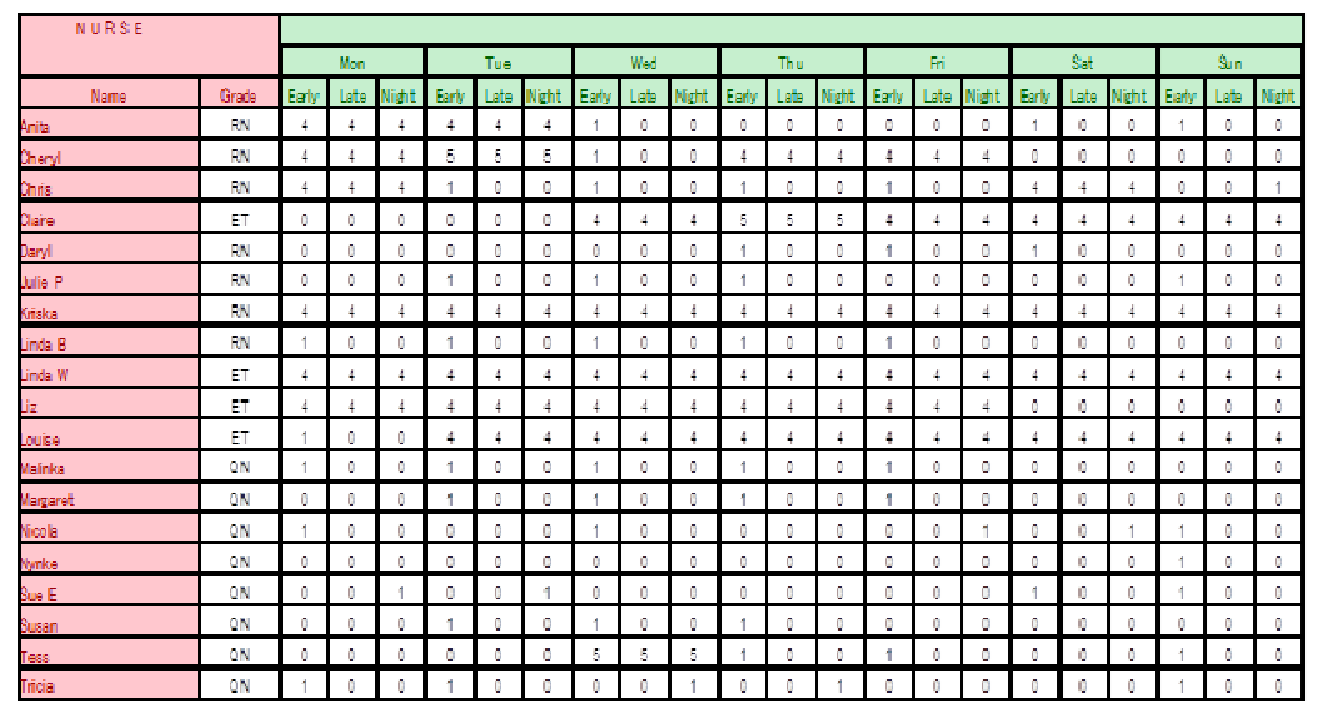




\section{Step 2: Fitness \&Selection}

The better fit solutions are selected using the tournament selection. The fitness function which acts as the criterion for selection is defined as follows: Penalties are imposed on the violations of the hard constraints (Table 4) and on the violations of the soft constraints, as well (Table 5).

Table 4. Penalties on the violations of the hard constraints

\begin{tabular}{|l|c|}
\hline \multicolumn{1}{|c|}{ Hard Constraints } & Penalty $p_{h i}$ \\
\hline OneShiftADay & 4000 \\
\hline MaxHours & 2000 \\
\hline MaxDaysOn & 3000 \\
\hline MinDaysOn & 1000 \\
\hline Succession & 6000 \\
\hline HardRequest & 5000 \\
\hline
\end{tabular}

Table 5. Penalties on the violations of the soft constraints

\begin{tabular}{|l|c|}
\hline \multicolumn{1}{|c|}{ Soft Constraints } & Penalty $p_{c i}$ \\
\hline SoftRequest & 4 \\
\hline SingleNight & 1 \\
\hline WeekendBalance & 2 \\
\hline WeekendSplit & 3 \\
\hline Coverage & 5 \\
\hline
\end{tabular}

The total penalties on the violations of the hard constraints are given by:

$f=\sum_{i=1}^{N} p_{h i} x_{i}$

The total penalties on the violations of the soft constraints are given by:

$$
g=\sum_{i=1}^{N} p_{c i} x_{i}
$$

The objective function is given by:

$$
\begin{aligned}
& \quad h=f+g \quad(3) \\
& x \in\{0,1\} \quad(i=1,2, \cdots, N-1, N)(4)
\end{aligned}
$$

Penalties are arbitrary and need not be an exact indicator of cost, time, etc. [13]. They can serve as fitness functions to guide the search. In our study, we consider the fitness function to be the same as the objective function and being a minimization problem, the solutions with lower values of fitness function are better than those with higher values. We have imposed arbitrary 1000 unit penalties on the violation of hard penalties and 1 unit on the violation of soft penalties. The motivation behind this is that, when the evolving solutions reach a fitness function value which is less than 1000 (lower value is better), it is an indication that all the hard constraints are being satisfied and the solutions are feasible.

\section{Step 3: Crossover}

We have tailored the crossover operator to suit the QMC NSP tabular representation. The $i^{\text {th }}$ row and the $\mathrm{j}^{\text {th }}$ column in the shifts table are randomly selected and used to demarcate the shifts tables 
in four parts as shown in Fig. 2. The offspring are obtained by interchanging the corresponding parts as shown in Fig. 3.
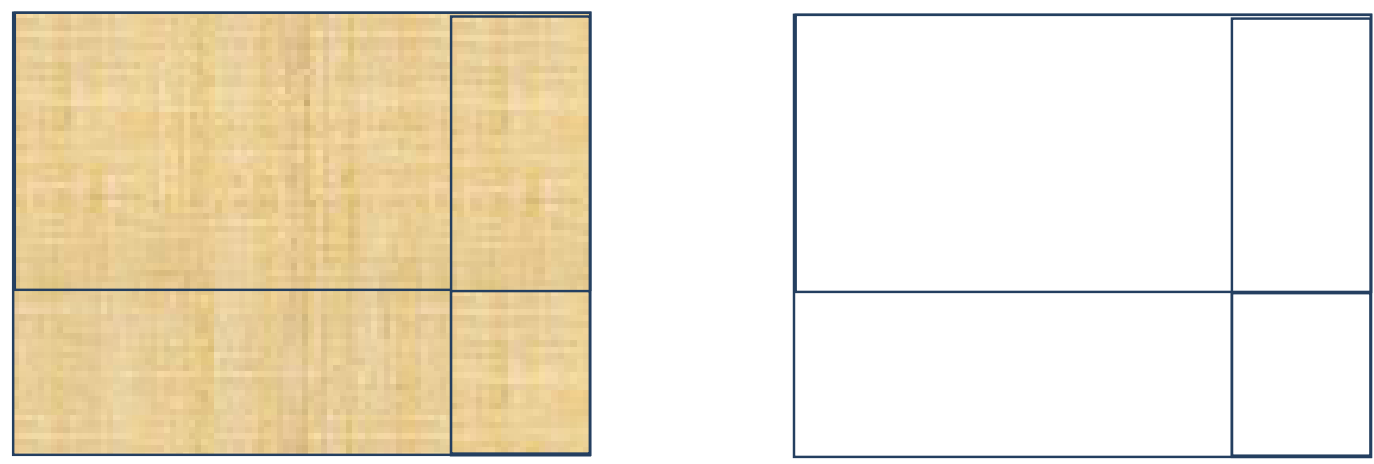

Fig. 2. Parents selected for crossover
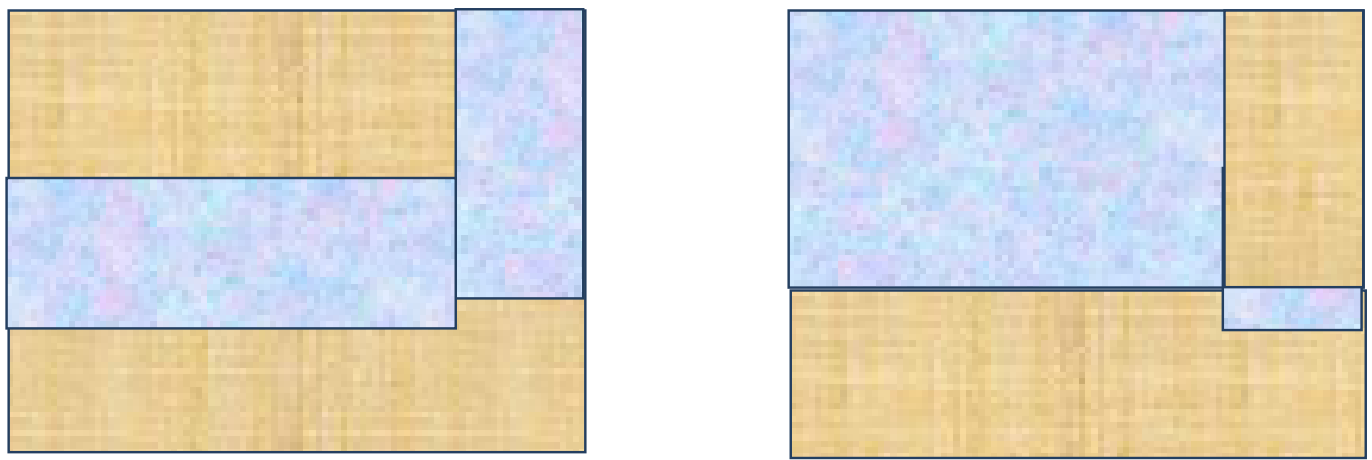

Fig. 3. Offspring obtained after crossover

\section{Step 4: Mutations}

Mutations are done by randomly selecting a bit and converting it into zero if it is non-zero and vice-versa.

\section{Step 5: Local Search}

We employ the following greedy Local Search approach which incorporates the intrinsic knowledge of the problem. The QMC NSP has six hard constraints which correspond to the managementrequirement. Any one of the six hard constraints are randomly imposed as explained below.

1.OneShiftADay: A nurse canwork only one shift per day

If any nurse has more than one shift assigned to her in a given day, the shift is altered so that she has no more than a single shift that day as shown in Fig. 4.

\begin{tabular}{|c|c|c|}
\hline Early & Late & Night \\
\hline 0 & 1 & 1 \\
\hline
\end{tabular}

\begin{tabular}{|c|c|c|}
\hline Early & Late & Night \\
\hline 0 & 0 & 1 \\
\hline
\end{tabular}

\begin{tabular}{|c|c|c|}
\hline Early & Late & Night \\
\hline 0 & 1 & 0 \\
\hline
\end{tabular}

Fig. 4. Local Search: One Shift A Day 
2. MaxHours: The nurses work a maximum of 40 hours a week

If any nurse is exceeds the maximum number of hours allowed per week, her shifts are randomly adjusted to reduce the number of hours as shown in Fig. 5.

\begin{tabular}{|c|c|c|c|c|c|c|c|c|c|c|c|c|c|c|c|c|c|c|}
\hline & \multicolumn{2}{|r|}{ Tue } & \multicolumn{2}{|r|}{ Wed } & \multicolumn{3}{|c|}{ Thu } & \multicolumn{2}{|r|}{ Fii } & & \multicolumn{2}{|r|}{ Sat } & & \multicolumn{3}{|c|}{ Sun } & \multirow{2}{*}{\begin{tabular}{|l|} 
Total \\
Time
\end{tabular}} \\
\hline Early L & Late Night & Early & Late Night & Eaty & Late Nigh & Earty & Late & Night_L & Earty & Late & Vight & Eaty & Late & Vight & Earty & Late & light & \\
\hline 1 & \begin{tabular}{l|l}
0 & 0 \\
\end{tabular} & \begin{tabular}{|l|}
0 \\
\end{tabular} & \begin{tabular}{|l|l}
0 & 1 \\
\end{tabular} & 0 & \begin{tabular}{|l|l}
0 & 1 \\
\end{tabular} & 0 & 0 & 0 & 1 & 0 & 0 & 1 & 0 & 0 & 0 & & 0 & 50 \\
\hline & & & & & & & & $\downarrow$ & & & & & & & & & & \\
\hline & Mon & & Tue & & Wed & & Thu & & & Fri & & & Sat & & & Sun & & Total \\
\hline Earty L. & Late Vight & Early & Late Night & Eaty & Late Night & Eaty & & Night| & Earty & Late & Night & Eath & Late & $\sqrt{\text { Vight }}$ & Earty & Late & igh & Tme \\
\hline 1 & \begin{tabular}{l|l}
0 & 0 \\
\end{tabular} & \begin{tabular}{|l|}
0 \\
\end{tabular} & \begin{tabular}{l|l}
0 & 1 \\
\end{tabular} & \begin{tabular}{|l|}
0 \\
\end{tabular} & \begin{tabular}{|l|l}
0 & 0 \\
\end{tabular} & $\begin{array}{l}0 \\
\end{array}$ & 0 & 0 & 1 & 0 & 0 & 1 & 0 & 0 & 0 & & 0 & 40 \\
\hline
\end{tabular}

Fig. 5. Local Search: Maximum hours a week

3.MaxDaysOn:(not more than six days in a row)

If any nurse is found to do more than the six days in a row, she is made to take a break in between as shown in Fig. 6.

\begin{tabular}{|c|c|c|c|c|c|c|c|}
\hline Sun & Mon & Tue & Wed & Thu & Fri & Sat & Sun \\
\hline 0 & 1 & 1 & 1 & 1 & 1 & 1 & 0 \\
\hline Sun & Mon & Tue & Wed & Thu & Fri & Sat & Sun \\
\hline 0 & 1 & 1 & 0 & 1 & 1 & 1 & 0 \\
\hline
\end{tabular}

Fig. 6. Local Search: Max Days On

4. MinDaysOn: (At least two days a week in succession)

If any nurse is found not to have a shift of at least two days in succession, her shift is altered as shown in Fig. 7.

\begin{tabular}{|c|c|c|}
\hline Wed & Thu & Fri \\
\hline 0 & 1 & 0 \\
\hline \multicolumn{3}{|c}{} \\
\end{tabular}

\begin{tabular}{|c|c|c|c|c|c|}
\hline Wed & Thu & Fri \\
\hline 0 & 1 & 1 & Wed & Thu & Fri \\
\hline
\end{tabular}$\quad$\begin{tabular}{c}
\hline 1 \\
\hline
\end{tabular}

Fig. 7. Local Search: Min Days On

5. Succession(No day shift after a night shift)

If any nurse is found to do a day shift immediately after a night shift or vice versa, her shift is altered as shown in Fig. 8. 
International Journal of Artificial Intelligence \& Applications (IJAIA) Vol. 6, No. 4, July 2015

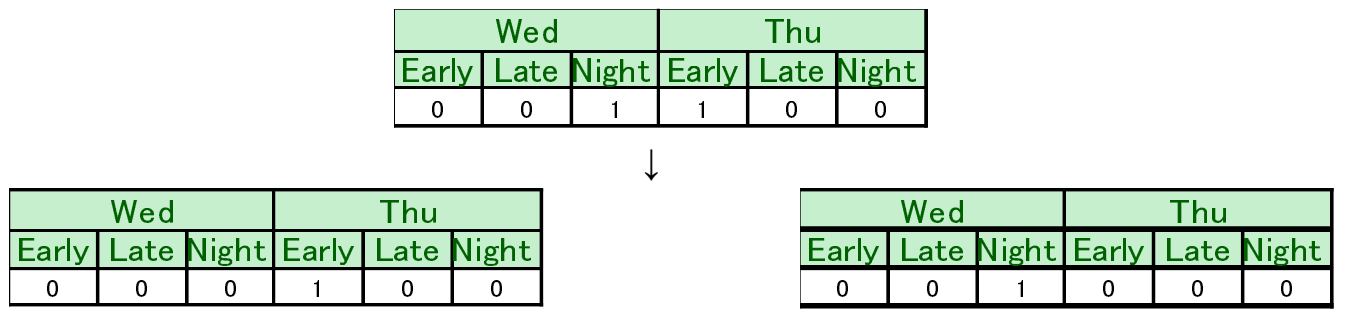

Fig. 8. Local Search: Succession

6. Hard Request (Nurses must take annual leave)

Nurses must take the annual leave they are entitled to. If nay nurse is found not to take the annual shift, her shifts are altered so that she can take the due annual leave as shown in Fig. 9.

\begin{tabular}{l|c|c|}
\hline Early & Late & Night \\
\hline 5 & 5 & 5 \\
\hline \multicolumn{3}{|c|}{$\downarrow$} \\
\hline Early & Late & Night \\
\hline 0 & 0 & 0 \\
\hline
\end{tabular}

Fig. 9. Local Search: Hard Request

The hybrid MA solves the NSP in two phases. In the first phase it tries to search for the nurses' shift patterns that do not violate the hard constraints. These solutions are indicated by a fitness value of less than 1000. In the second phase, the MA further evolves these feasible solutions to minimize the penalties on the soft constraints. One of the optimal solutions to the QMC NSP is shown in Table 6.

Table 6. A shifts schedule optimized using MA

\begin{tabular}{|c|c|c|c|c|c|c|c|c|c|c|c|c|c|c|c|c|c|c|c|c|c|c|c|c|c|c|c|c|}
\hline & 5 & 6 & ] & 8 & 3 & 10 & 11 & 12 & 13 & 14 & 15 & 16 & 17 & 18 & 12 & 20 & 21 & 22 & 23 & 24 & 25 & 26 & 27 & 28 & 23 & 30 & 31 & I \\
\hline & I. & 7 & 品 & $\mathrm{th}$ & $\vdots$ & $s$ & Sy & $\mathrm{E}$ & 7 & $\mathrm{~g}$ & $\mathrm{z}$ & $\vdots$ & $\$$ & St & $\mathbb{E}$ & 7 & $\mathrm{z}$ & $\mathrm{th}$ & $\bar{z}$ & 3 & So & $\mathrm{E}$ & 7 & $\mathrm{~g}$ & $\mathrm{n}$ & $\Xi$ & 3 & Sh \\
\hline mita & $z$ & I & $\Xi$ & E & I & & & $z$ & I & I & $z$ & $\mathrm{E}$ & & & $\mathrm{AL}$ & $\mathrm{IL}$ & IIL & II & AL & 2IL & AL & $\mathrm{AL}$ & AL & I & I & $\pi$ & $\pi$ & \\
\hline Saryl & & $\mathrm{AL}$ & L & L & $z$ & & & & II & $z$ & fI. & 8 & & & $\mathrm{E}$ & $\mathrm{H}$ & EI & II & AL & KL & II & $=$ & L & & 8 & $z$ & & \\
\hline "hris & QII & $\mathrm{Z}$ & I & $z$ & & & $\pi$ & & I & I & IL & I & 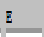 & & & & $\mathrm{E}$ & & & & & & & $z$ & & & & \\
\hline Plaire & & E & $z$ & $\mathrm{EI}$ & $z$ & & & $z$ & I & $z$ & FI & FI & & & 2 & $z$ & $z$ & & & $z$ & $z$ & 2 & E & $z$ & & & I & $z$ \\
\hline aryl & & & & $\Xi$ & $\Xi$ & $z$ & I & I & & I & II & II & AL & $\pi$ & & & $z$ & & I & $\Xi$ & AL & 2L & AI & IL & AL & $\mathrm{AL}$ & LL & AL \\
\hline mis ? & & E & L & Z & & & 5 & & & $\pi$ & II & & & & & z & L & Z & & & I & & $=$ & $E$ & AI & & & \\
\hline riska & 8 & I & $\mathbb{Z}$ & & I & $\mathbb{Z}$ & $z$ & & I & E & I & $\mathbb{Z}$ & & & $\mathrm{AL}$ & II & II & $\mathbb{I I}$ & AL & II & LL & $\mathbb{L}$ & $\mathrm{AL}$ & $\mathbb{Z}$ & & & $\mathbb{Z}$ & I \\
\hline Enda 3 & E & 2 & E & L & $E$ & & & T. & $\pi$ & & L & AL & & & E & I & & & L & E & I & $=$ & $E$ & I & & & $z$ & L \\
\hline Lisia $\mathrm{g}$ & $L$ & I & & & 8 & I & & & 8 & & 8 & & I & 8 & $\mathbb{L}$ & IL & II & $\mathbb{L}$ & $\mathrm{AL}$ & & & & & I & I & & 8 & I \\
\hline 12 & & 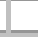 & & $z$ & $z$ & & & 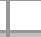 & & & & & $z$ & L & & & & $z$ & $E$ & & & & & & 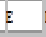 & $E$ & & \\
\hline wouise & I & $\Xi$ & $\Xi$ & I & $\Xi$ & & & I & $\Xi$ & $z$ & & & E & I & I & $\Xi$ & $\mathrm{E}$ & I & $\Xi$ & & & $\mathbb{\pi}$ & $\pi$ & $\mathbb{I}$ & $\mathbb{\pi}$ & & & \\
\hline balinka & $=$ & - & $z$ & L & 2 & & & $z$ & 2 & I & & & I & 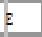 & $z$ & I & 8 & & & L & $z$ & $=$ & 8 & I & 8 & AL & $\mathrm{H}$ & AL \\
\hline targaret & & $\Xi$ & I & I & $\Xi$ & & & I & $\Xi$ & I & & & & I & $z$ & I & $\pi$ & $\mathbb{\pi}$ & & & & E & I & & I & $z$ & & \\
\hline Rionla & $z$ & & $z$ & & $\pi$ & & $z$ & D & & & & L & & 8 & $z$ & & & & L & & $z$ & $z$ & & & & E & & z \\
\hline Jyke & & & & & & & I & $z$ & I & & & & & & $\mathbb{I}$ & $\mathbb{\pi}$ & & & & & & & & & & & & \\
\hline ian: & h & $n$ & & & & 8 & $z$ & : & : & I & 8 & & & & : & : & E. & I & 8 & & & : & E & E & & & I & 8 \\
\hline Susan & & I & E & E & & & & AI & $\mathrm{BL}$ & II & & & & & $\mathrm{kI}$ & & & & E & E & E & $\mathbb{1}$ & $\mathbb{I}$ & & & & & \\
\hline
\end{tabular}

\section{Conclusion}

The Nurse Scheduling Problem (NSP), like the well-known Travelling Salesman Problem (TSP), is an NP-hard problem. Some studies show that the straightforward implementation of the 
Genetic Algorithm is incapable of obtaining a satisfactory solution. Therefore, we believe that a hybrid methodology involving an Evolutionary Algorithm that finds several feasible solutions and a Local Search exploiting the inherent knowledge of the problem to optimize the intermediate feasible solutions is an appropriate tool to tackle this highly complex problem. With this intuition, we have tailor-made a Memetic Algorithm (GA + local search) to solve the Queen's Medical Centre (Nottingham, UK) NSP that is freely available online. The hybrid MA solves the NSP in two phases. In the first phase it tries to search for the nurses' shift patterns that do not violate the hard constraints. In the second phase, the MA further evolves these feasible solutions to minimize the penalties on the soft constraints and obtains optimal and near optimal solutions.

\section{References}

[1] Abobaker, R.A.; Ayob, M.; Hadwan, M., "Greedy constructive heuristic and local search algorithm for solving Nurse Rostering Problems," 3rd Conference on Data Mining and Optimization (DMO), 2011, pp.194,198, 28-29 June 2011.

[2] Aickelin U and Dowsland KA. Exploiting problem structure in a Genetic Algorithms approach to a nurserostering problem. Journal of Scheduling, vol. 31, pp.139-153, 2000.

[3] Uwe Aickelin and Kathryn A. Dowsland, An Indirect Genetic Algorithm for a Nurse Scheduling ProblemComputers \& Operations Research, 31(5), pp. 761-778, 2004.

[4] Aickelin, U.; Burke, E.K.; Jingpeng Li, "An Evolutionary Squeaky Wheel Optimization Approach to Personnel Scheduling," IEEE Transactions on Evolutionary Computation, , vol.13, no.2, pp.433,443, April 2009.

[5] AlpayAlkan, Ender Özcan, Memetic Algorithms for Timetabling, Evolutionary Computation, EC 2003, pp. $1796-1802$. Vol.3

[6] Bai, R.; Burke, E.K.; Kendall, G.; Jingpeng Li; McCollum, B., "A Hybrid Evolutionary Approach to the Nurse Rostering Problem," IEEE Transactions on Evolutionary Computation, , vol.14, no.4, pp.580,590, Aug. 2010.

[7] Edmund K. Burke,Patrick deCausmaecker, Greet VandenBerghe and Hendrik Van Landeghem, The state of the art of Nurse Rostering, JournalofScheduling7: 441-499, 2004.

[8] Cares, J.P.; Riff, M.C., "A Collaborative Approach to Solve a Nurse Scheduling Problem," 24th IEEE International Conference on Tools with Artificial Intelligence (ICTAI), 2012, vol.1, pp.344,350, 7-9 Nov. 2012.

[9] Charles W. Spry and Mark A. Lawley, "Evaluating Hospital Pharmacy Staffing and Work Scheduling using Simulation, Proceedings of the 2005 Winter Simulation Conference,M. E. Kuhl, N. M. Steiger, F. B. Armstrong, and J. A. Joines, eds.pp. 2256-2263.

[10] Mingang Cheng, Hiromi Itoh Ozaku, NoriakiKuwahara, Kiyoshi Kogure and Jun Ota, "Nursing Care Scheduling Problem: Analysis ofStaffing Levels", Proceedings of the 2007 IEEEInternational Conference on Robotics and Biomimetics, December 15 -18, 2007, Sanya, China, pp. 1715-1720.

[11] Constantino, A.A.; Landa-silva, D.; de Melo, E.L.; Romao, W., "A heuristic algorithm for nurse scheduling with balanced preference satisfaction," IEEE Symposium on Computational Intelligence in Scheduling (SCIS), 2011, pp.39,45, 11-15 April 2011.

[12] Davis, L. (1991). Handbook of genetic algorithms. New York: Van Nostrand Reinhold.

[13] K. Deb, Genetic algorithms for function optimization J.L. Herraand, L. Verdegay (Eds.), Genetic Algorithms and Soft Computing, Physica-Verlag, Heidelberg (1996).

[14] Dowsland KA. Nurse Scheduling with Tabu Search and Strategic Oscillation. European Journal ofOperational Research 1998; 106:393-407.

[15] Dowsland KA and Thompson JM. Nurse Scheduling with Knapsacks, Networks and Tabu Search.Journal of the Operational Research Society 2000; 825-833.

[16] Easton F and Mansour N. A Distributed Genetic Algorithm for Employee Staffing and SchedulingProblems. In Forrest S. Editor. Proceedings of the Fifth International Reference on Genetic Algorithms,San Mateo: Morgan Kaufmann Publishers, 1993:360-367.

[17] Elbenani, B.; Ferland, J.A.; Gascon, V., "Mathematical programming approach for routing home care nurses," IEEE International Conference on Industrial Engineering and Engineering Management, 2008. IEEM 2008, pp.107,111, 8-11 Dec. 2008.

[18] Goldberg, D. E., and Voessner, S. (1999). Optimizing global-local search hybrids. In W. Banzhafet al. (Eds.), Proceedings of the Genetic and Evolutionary Computation Conference' 99 (pp. 220-228). San Mateo, CA: Morgan Kaufmann. 
International Journal of Artificial Intelligence \& Applications (IJAIA) Vol. 6, No. 4, July 2015

[19] Hadwan, M.; Ayob, M., "A constructive shift patterns approach with simulated annealing for nurse rostering problem," 2010 International Symposium in Information Technology (ITSim), , vol.1, pp.1,6, 15-17 June 2010.

[20] Hasegawa, S.; Kosugi, Yukio, Solving Nurse Scheduling Problem by Integer-Programming-Based Local Search," IEEE International Conference on Systems, Man and Cybernetics, 2006. SMC '06., vol.2, pp.1474,1480, 8-11 Oct. 2006.

[21] Hung R. Hospital Nurse Scheduling. Journal of Nursing Administration 1995; 1:21-23.

[22] Inoue, T.; Furuhashi, T.; Fujii, M.; Maeda, H.; Takaba, M., "Development of nurse scheduling support system using interactive EA," 1999 IEEE International Conference on Systems, Man, and Cybernetics, 1999. vol.5, pp.533,537, 1999.

[23] Inoue, T.; Furuhashi, T.; Maeda, H.; Takaba, M., "A proposal of combined method of evolutionary algorithm and heuristics for nurse scheduling support system," IEEE Transactions on Industrial Electronics,vol.50, no.5, pp.833,838, Oct. 2003.

[24] Kawanaka, H.; Yamamoto, K.; Yoshikawa, T.; Shinogi, T.; Tsuruoka, S., "Genetic algorithm with the constraints for nurse scheduling problem," Proceedings of the 2001 Congress on Evolutionary Computation, 2001., vol.2, pp.1123-1130.

[25] Krasnogor, N., and Smith J. E. (2001). Emergence of profitable search strategies based on a simple inheritance mechanism. In Proceedings of the 2001 International Conference on Genetic and Evolutionary Computation, pp. 432-439. San Mateo, CA: Morgan Kaufmann.

[26] Krasnogor, N., and Smith, J. E. (2005). A tutorial for competent memetic algorithms: Model, taxonomy, and design issue. IEEE Transactions on Evolutionary Computation, 9(5):474-488.

[27] Khoi Nguyen Le , QMC Nurse Scheduling Problem, Univiversity of Nottigham . http://www.cs.nott.ac.uk/ kxl/research/QMC/QMC.pdf; accessed on Dec. 1, 2010.

[28] Lo, C.-C.; Lin, C.-C.; Wang, C.-T.; Dai, T.-J.; Wong, D., "Artificial immune systems for intelligent nurse rostering," 2007 IEEE International Conference on Industrial Engineering and Engineering Management, pp.862,866, 2-4 Dec. 2007.

[29] Merz, P. (2000).Memetic algorithms for combinatorial optimization problems: Fitness landscapes and effective search strategies. Ph.D. Thesis, University of Siegen, Germany.

[30] Mingang Cheng; Ozaku, H.I.; Kuwahara, N.; Kogure, K.; Ota, J., "Simulated annealing algorithm for scheduling problem in daily nursing cares," IEEE International Conference on Systems, Man and Cybernetics, 2008. SMC 2008., pp.1681,1687, 12-15 Oct. 2008.

[31] D. Molina, M. Lozano, C. García-Martínez, and F. Herrera, Memetic Algorithms for Continuous Optimisation Based on Local Search Chains, Evolutionary Computation 18(1): 27-63.

[32] Moscato, P. A. (1999). Memetic algorithms: A short introduction. In D. Corne, M. Dorigo, andF. Glower (Eds.), New ideas in optimization (pp. 219-234). New York: McGraw-Hill.

[33] Q. H. Nguyen, Y. S. Ong, and N. Krasnogor, A Study on the Design Issues of Memetic Algorithm, IEEE Congress on Evolutionary Computation, CEC 2007, pp. 2390 - 2397.

[34] Ohki, M.; Morimoto, A.; Miyake, K., "Nurse Scheduling by Using Cooperative GA with Efficient Mutation and Mountain-Climbing Operators," 3rd International IEEE Conference on Intelligent Systems, 2006, pp.164,169, Sept. 2006.

[35] Ohki, M.; Uneme, S.; Kawano, H., "Parallel processing of cooperative genetic algorithm for nurse scheduling," 4th International IEEE Conference on Intelligent Systems, 2008. IS '08., vol.2, pp.10-36, 6-8 Sept. 2008

[36] Ohki, M.; Kinjo, H., "Penalty weight adjustment in cooperative GA for nurse scheduling," Third World Congress on Nature and Biologically Inspired Computing (NaBIC), 2011, . pp.75,80, 19-21 Oct. 2011.

[37] Oughalime, A.; Ismail, W.R.; LiongChoongYeun, "A Tabu Search approach to the nurse scheduling problem," International Symposium on Information Technology, 2008. ITSim 2008. , vol.1, pp.1,7, 26-28 Aug. 2008.

[38] Sitompul D and Randhawa S. Nurse Scheduling Models: A State-of-the-Art Review. Journal of theSociety of Health Systems 1990; 2:62-72.

[39] Tanomaru J. Staff Scheduling by a Genetic Algorithm with Heuristic Operators. Proceedings of the IEEEReference on Evolutionary Computation 1995; 456-461.

[40] Zen-El-Din, W.K.; Kamel, M.S.A.; Mohamed, A.S.A., "A binary integer programming for nurse scheduling," 2012 Cairo International Biomedical Engineering Conference (CIBEC), pp.122,125, 2022 Dec. 2012. 